\title{
Improved Analysis for Squeezing of Newtonian Material between Two Circular Plates
}

\author{
Omar Khan, ${ }^{1}$ Mubashir Qayyum, ${ }^{2}$ Hamid Khan, ${ }^{2}$ and Murtaza Ali ${ }^{3}$ \\ ${ }^{1}$ Department of Computer Science, National University of Computer \& Emerging Sciences, Peshawar, Pakistan \\ ${ }^{2}$ Department of Mathematics, National University of Computer \& Emerging Sciences, Peshawar, Pakistan \\ ${ }^{3}$ Department of Mathematics, University of Engineering \& Technology, Mardan, Pakistan \\ Correspondence should be addressed to Omar Khan; omar.khan@nu.edu.pk
}

Received 4 September 2016; Revised 7 March 2017; Accepted 13 March 2017; Published 28 March 2017

Academic Editor: Wenbin Yi

Copyright (C) 2017 Omar Khan et al. This is an open access article distributed under the Creative Commons Attribution License, which permits unrestricted use, distribution, and reproduction in any medium, provided the original work is properly cited.

This article presents a scheme for the analysis of an unsteady axisymmetric flow of incompressible Newtonian material in the form of liquid squeezed between two circular plates. The scheme combines traditional perturbation technique with homotopy using an adaptation of the Laplace Transform. The proposed method is tested against other schemes such as the Regular Perturbation Method (RPM), Homotopy Perturbation Method (HPM), Optimal Homotopy Asymptotic Method (OHAM), and the fourth-order Explicit Runge-Kutta Method (ERK4). Comparison of the solutions along with absolute residual errors confirms that the proposed scheme surpasses HPM, OHAM, RPM, and ERK4 in terms of accuracy. The article also investigates the effect of Reynolds number on the velocity profile and pressure variation graphically.

\section{Introduction}

The study of squeezing flows has significant applications in the areas of engineering, physics, biology, and material sciences. In the past few years, the study of rheometric properties of fluids has garnered significant attention due to its vast industrial applications. Examples include modelling of lubrication systems involved in squeezing of fluids [1-3], compression moulding processes of metals and polymers [4], injection moulding processes, polymer processes [5], hydrodynamical tools and machines, modelling of chewing and eating [6], and modelling of the functions of heart valves and blood vessels. Many of these applications involve the adjustment of rheometric properties using external stimuli such as electric and magnetic fields. For instance, electrorheological fluids (micron size polymer particles in silicon) may solidify or become extremely viscous under an electric field. The same can be said about magnetorheological fluids involving magnetic particles. Under an applied external field, these particles remain suspended due to which fluid particles are not able to exhibit Brownian motion. As a result, the fluid can adopt viscous properties.
Traditional approaches to study flow patterns involve the configuration of two plates of radius $\alpha$ that are separated by a narrow gap $h(t)$. Three modes of operations on the plates are commonly used; stationary plates resulting in Poiseuille flow, shear mode resulting in Couette flow, and squeeze mode resulting in compressed flow. Some properties of the flow such as mass and momentum are not affected by deformations due to these operations and they remain conserved. The resulting set of properties such as velocity and pressure can be modelled as various boundary value problems. Some configurations may also focus on the interaction between the samples and the plates. The interactions can result in different types of stresses identified as slip, no-slip, or partial slip. Other configurations may also focus on types of fluid such as viscous, plastics (or viscoplastics), and elastic (or viscoelastic) fluids.

The solutions to these different configurations and boundary value problems can be obtained using well known analytical [7-14] and numerical schemes [15, 16]. The most common approach involves the usage of perturbation techniques that assume small or large parameters, which may affect the solutions in different scenarios. To overcome this 


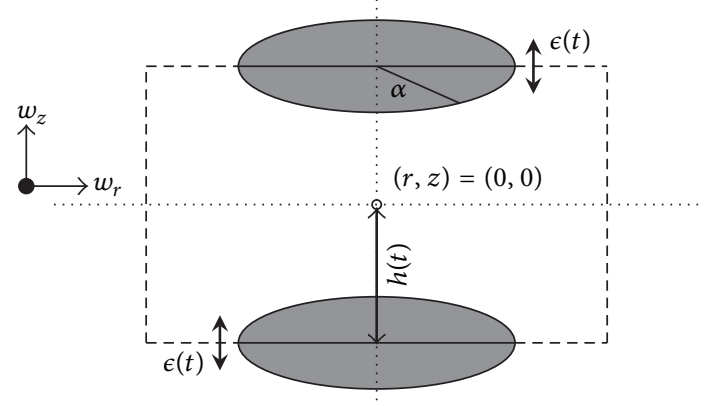

FIGURE 1: Geometrical interpretation of squeezing flow.

limitation, a method known as the Homotopy Perturbation Method was introduced that combined traditional perturbations with homotopy and was applied to various nonlinear boundary value problems [7, 8, 17-19]. Some modifications and extensions to the method have also appeared. Examples include that of dynamical systems of rotating machines [20], nonlinear differential equations [21], singular lane-embed equations [22], Cauchy reaction diffusion equation [23], nonlinear undamped oscillators [24], and fractional differential equations [25].

In this article, we present a detailed analysis of squeezing flow of Newtonian fluids using an improved alteration of the Homotopy Perturbation Method with Laplace Transform. We refer to this alteration as HPLM, that is, Homotopy Perturbation Laplace Method. To check the effectiveness of the proposed scheme, a comparison is performed with Regular Perturbation Method (RPM), Homotopy Perturbation Method (HPM), Runge-Kutta (Explicit) method of fourth-order, and Optimal Homotopy Asymptotic Method (OHAM). Moreover, the effect of Reynolds number on the velocity profile and pressure variation is studied graphically.

\section{Mathematical Formulation}

The formulation is based on an unsteady squeezing flow of an incompressible Newtonian fluid bearing kinematic viscosity $\nu$, density $\rho$, and viscosity $\mu$. The fluid is squeezed between a pair of circular plates that are at a distance of $2 h(t)$. The plates operate in a squeezing mode where the plate velocity is $\epsilon(t)$. A 2 D configuration of squeezing flow in $(z, r)$ plane is in Figure 1, where the plates move perpendicular to the central axis $z=0$. With this operation, an axisymmetric behaviour is observed in the flow about $r=0$. The velocity components (normal and longitudinal) in axial and radial directions are $w_{z}(z, r, t)$ and $w_{r}(z, r, t)$. The governing equations are

$$
\begin{gathered}
\frac{\partial w_{r}}{\partial r}+\frac{w_{r}}{r}+\frac{\partial w_{z}}{\partial z}=0 \\
\frac{\partial \widehat{P}}{\partial r}+\rho\left(\frac{\partial w_{r}}{\partial t}-w_{z} \Omega\right)=0-\mu \frac{\partial \Omega}{\partial z}, \\
\frac{\partial \widehat{P}}{\partial z}+\rho\left(\frac{\partial w_{z}}{\partial t}+w_{r} \Omega\right)=\frac{\mu}{r} \frac{\partial}{\partial r}(r \Omega) .
\end{gathered}
$$

Two kinds of boundary conditions are placed for the velocity components of the fluid. The first is due to no-slip at the upper plate, while the second is due to symmetry.

$$
\begin{aligned}
w_{r}(z, r, t) & =0, \\
w_{z}(z, r, t) & =v_{w}(t), \\
\frac{\partial}{\partial z} w_{r}(z, r, t) & =0, \\
w_{z}(z, r, t) & =0,
\end{aligned}
$$

for $z=0$.

By introducing the dimensionless parameter $\eta=z / h(t),(1)$, (2), and (3) are transformed to

$$
\begin{gathered}
\frac{\partial w_{r}}{\partial r}+\frac{w_{r}}{r}+\frac{1}{h} \frac{\partial w_{z}}{\partial \eta}=0 \\
\frac{\partial \widehat{P}}{\partial r}+\rho\left(\frac{\partial w_{r}}{\partial t}-w_{z} \Omega\right)=-\frac{\mu}{h} \frac{\partial \Omega}{\partial \eta}, \\
\frac{1}{h} \frac{\partial \widehat{P}}{\partial \eta}+\rho\left(\frac{\partial w_{z}}{\partial t}+w_{r} \Omega\right)=\frac{\mu}{r} \frac{\partial}{\partial r}(r \Omega),
\end{gathered}
$$

where the boundary conditions are

$$
\begin{aligned}
w_{r} & =0, \\
w_{z} & =\epsilon(t), \\
& \text { for } \eta=1, \\
\frac{\partial w_{r}}{\partial \eta} & =0, \\
w_{z} & =0,
\end{aligned}
$$

$$
\text { for } \eta=0
$$

After eliminating the generalized pressure between (6) and (7), the following is obtained:

$$
\rho\left[\frac{\partial \Omega}{\partial t}+w_{r} \frac{\partial \Omega}{\partial r}+\frac{w_{z}}{h} \frac{\partial \Omega}{\partial \eta}-\frac{w_{r}}{r} \Omega\right]=\mu\left[\nabla^{2} \Omega-\frac{\Omega}{r^{2}}\right],
$$

where $\nabla^{2}$ is the Laplacian operator.

Defining velocity components as [4]

$$
\begin{aligned}
& w_{r}=-\frac{r \epsilon(t)}{2 h(t)} G^{\prime}(\eta), \\
& w_{z}=\epsilon(t) G(\eta),
\end{aligned}
$$

(5) is identically satisfied, while (9) becomes

$$
\frac{\mathrm{d}^{4} G}{\mathrm{~d} \eta^{4}}+R\left[(\eta-G) \frac{\mathrm{d}^{3} G}{\mathrm{~d} \eta^{3}}+2 \frac{\mathrm{d}^{2} G}{\mathrm{~d} \eta^{2}}\right]-Q \frac{\mathrm{d}^{2} G}{\mathrm{~d} \eta^{2}}=0
$$


where

$$
\begin{aligned}
& R=\frac{h \epsilon}{\nu}, \\
& Q=\frac{h^{2}}{\nu \epsilon} \frac{\mathrm{d} \epsilon}{\mathrm{d} t} .
\end{aligned}
$$

Integrating first equation of (12) gives

$$
h(t)=\sqrt{c t+d},
$$

where $c$ and $d$ are constants affecting the directional movement of the plates. From (12) and (13), it follows that $Q=-R$. Now, (11) becomes

$$
\frac{\mathrm{d}^{4} G}{\mathrm{~d} \eta^{4}}+R\left[(\eta-G) \frac{\mathrm{d}^{3} G}{\mathrm{~d} \eta^{3}}+3 \frac{\mathrm{d}^{2} G}{\mathrm{~d} \eta^{2}}\right]=0
$$

Using (8) and (10), the boundary conditions in case of no-slip at the upper plate are

$$
\begin{aligned}
G(1) & =1, \\
G^{\prime}(1) & =0, \\
G(0) & =0, \\
G^{\prime \prime}(0) & =0 .
\end{aligned}
$$

Furthermore, using (10) in (3) and integrating with respect to $\eta$ while keeping $r$ fixed, the variation of pressure is

$$
\begin{aligned}
& P(\eta, t)-P_{c} \\
& \quad=\rho \epsilon^{2}\left[\eta G-\frac{G^{2}}{2}+\frac{1}{R}\left(G^{\prime}(\eta)-G^{\prime}(0)\right)\right],
\end{aligned}
$$

where $P_{c}$ is the pressure at $\eta=0$. Similarly, using (10) in (2) and integrating with respect to $r$ while keeping $\eta$ fixed lead to the pressure distribution.

\section{Basic Theory of HPLM}

The basic concept of HPLM can be understood by applying it to the following differential equation:

$$
\mathfrak{J}[u(x)]+\aleph[u(x)]-f(x)=0,
$$

where $\mathfrak{J}$ and $\aleph$ are linear and nonlinear operators, while $f(x)$ and $u(x)$ are known and unknown functions. According to HPLM, a homotopy can be constructed as $u(x, p): \mathbb{R} \times$ $[0,1] \rightarrow \mathbb{R}$ such that it satisfies

$$
\begin{aligned}
(p-1) & {[f(x)-\mathfrak{J}(u(x, p))] } \\
- & p[f(x)-\mathfrak{J}(u(x, p))-\aleph(u(x, p))]=0,
\end{aligned}
$$

where $p \in[0,1]$ is an embedding parameter while $x \in \mathbb{R}$. Expanding $u(x, p)$ using a Taylor series about $p$, we obtain an approximate solution:

$$
u(x, p)=u_{0}(x)+\sum_{k=1}^{m} u_{k} p^{k}
$$

Various order problems can be obtained by substituting (19) into (18) and equating with the coefficients of $p$. The zerothorder problem would be

$$
\mathfrak{J}\left[u_{0}(x)\right]-f(x)=0 .
$$

Applying Laplace Transform to (20) gives

$$
\begin{gathered}
s^{n} L u_{0}(x)-s^{n-1} u_{0}(\alpha)-s^{n-2} u_{0}^{\prime}(\alpha)-\cdots-u_{0}^{n-1}(\alpha) \\
-L\{f(x)\}=0 .
\end{gathered}
$$

Application of the inverse Laplace Transform to (21) gives

$$
\begin{aligned}
& u_{0}(x)=L^{-1}\left\{\frac { 1 } { s ^ { n } } \left[s^{n-1} u_{0}(\alpha)+s^{n-2} u_{0}^{\prime}(\alpha)+\cdots\right.\right. \\
& \left.\left.+u_{0}^{n-1}(\alpha)+L\{f(x)\}\right]\right\}
\end{aligned}
$$

The general $k$ th order problem would be

$$
\begin{aligned}
\mathfrak{J}\left[u_{k}(x)\right]-\aleph_{k-1}\left[u_{0}, u_{1}, \ldots, u_{k-1}\right]=0, & \\
& k=1,2,3, \ldots .
\end{aligned}
$$

Applying Laplace Transform to (23)

$$
\begin{gathered}
s^{n} L u_{k}(x)-s^{n-1} u_{k}(\alpha)-s^{n-2} u_{k}^{\prime}(\alpha)-\cdots-u_{k}^{n-1}(\alpha) \\
-L\left\{\aleph_{k-1}\left[u_{0}, u_{1}, \ldots, u_{k-1}\right]\right\}=0 .
\end{gathered}
$$

Application of the inverse Laplace Transform to (24) gives

$$
\begin{aligned}
& u_{k}(x)=L^{-1}\left\{\frac { 1 } { s ^ { n } } \left[s^{n-1} u_{k}(\alpha)+s^{n-2} u_{k}^{\prime}(\alpha)\right.\right. \\
& +s^{n-3} u_{k}^{\prime \prime}(\alpha)+\cdots+u_{k}^{n-1}(\alpha) \\
& \left.\left.+L\left\{\aleph_{k-1}\left[u_{0}, u_{1}, \ldots, u_{k-1}\right]\right\}\right]\right\}
\end{aligned}
$$

Using the boundary conditions, the approximate solution will be

$$
\widetilde{U}=\lim _{p \rightarrow 1} u=u_{0}+u_{1}+u_{2}+\cdots .
$$

Substituting (26) into (17), the expression for residual can be obtained as

$$
\text { Residual error }=\mathfrak{J}[\widetilde{U}(x)]+\aleph[\widetilde{U}(x)]-f(x) .
$$

The approach defined in this section minimizes the limitations of the ordinary perturbation methods and, in contrast, can take full advantage of the traditional perturbation techniques.

\section{Application of HPLM}

Using (11) and (15), various order problems are presented with their solutions in this section. The zeroth-order problem is

$$
u_{0}^{i v}(\eta)=0
$$


with boundary conditions $u_{0}(0)=0, u_{0}^{\prime}(0)=0, u_{0}^{\prime \prime}(0)=A$, and $u_{0}^{\prime \prime \prime}(0)=B$. The solution to $(28)$ is given as

$$
u_{0}(\eta)=A \eta+\frac{B \eta^{3}}{6}
$$

The first-order problem is

$$
u_{1}^{i v}(\eta)=-3 R u_{0}^{\prime \prime}(\eta)-R \eta u_{0}^{\prime \prime \prime}(\eta)+R u_{0}(\eta) u_{0}^{\prime \prime \prime}(\eta) .
$$

For (30) and the rest of higher order problems $u_{k}^{i v}(\eta)$, the boundary conditions are set as $u_{k}(0)=0, u_{k}^{\prime}(0)=0, u_{k}^{\prime \prime}(0)=$ 0 , and $u_{k}^{\prime \prime \prime}(0)=0$, where $k=1,2,3, \ldots$. The solution to (30) is given as

$$
u_{1}(\eta)=\frac{1}{120}(-4 B R+A B R) \eta^{5}+\frac{B^{2} R}{5040} \eta^{7} .
$$

The second-order problem is

$$
\begin{aligned}
u_{2}^{i v}(\eta)= & -3 R u_{1}^{\prime \prime}(\eta)+R u_{1}(\eta) u_{0}^{\prime \prime \prime}(\eta)-R \eta u_{1}^{\prime \prime \prime}(\eta) \\
& -R u_{0}(\eta) u_{1}^{\prime \prime \prime}(\eta)
\end{aligned}
$$

for which the solution is given as

$$
\begin{aligned}
u_{2}(\eta)= & \frac{1}{1680}\left(8 B R^{2}-6 A B R^{2}+A^{2} B R^{2}\right) \eta^{7} \\
& +\frac{1}{90720}\left(-13 B^{2} R^{2}+4 A B^{2} R^{2}\right) \eta^{9} \\
& +\frac{B^{3} R^{2}}{1108800} \eta^{11} .
\end{aligned}
$$

The third-order problem is

$$
\begin{aligned}
u_{3}^{i v}(\eta)= & -3 R u_{2}^{\prime \prime}(\eta)+R u_{2}(\eta) u_{0}^{\prime \prime \prime}(\eta) \\
& +R u_{1}(\eta) u_{1}^{\prime \prime \prime}(\eta)-R \eta u_{2}^{\prime \prime \prime}(\eta) \\
& +R u_{0}(\eta) u_{2}^{\prime \prime \prime}(\eta),
\end{aligned}
$$

for which the solution is given as

$$
\begin{aligned}
& u_{3}(\eta)=\left[\frac{B R^{3}}{120960}\left(-64+88 A-38 A^{2}+5 A^{3}\right) \eta^{9}\right. \\
& +\frac{B^{2} R^{3}}{39916800}\left(1720-1340 A+241 A^{2}\right) \eta^{11} \\
& +\frac{B^{3} R^{3}}{3113510400}(-2750+923 A) \eta^{13} \\
& \left.+\frac{1051 B^{4} R^{3}}{217945728000} \eta^{15}\right] .
\end{aligned}
$$

The fourth-order problem is

$$
\begin{aligned}
u_{4}^{i v}(\eta)= & -3 R u_{3}^{\prime \prime}(\eta)+R u_{3}(\eta) u_{0}^{\prime \prime \prime}(\eta) \\
& +R u_{2}(\eta) u_{1}^{\prime \prime \prime}(\eta)+R u_{1}(\eta) u_{2}^{\prime \prime \prime}(\eta) \\
& -R \eta u_{3}^{\prime \prime \prime}(\eta)+R u_{0}(\eta) u_{3}^{\prime \prime \prime}(\eta),
\end{aligned}
$$

to which the solution is given as

$$
\begin{aligned}
& u_{4}(\eta)=\left[\frac { B R ^ { 4 } } { 1 3 3 0 5 6 0 0 } \left(640-132888 A+996 A^{2}\right.\right. \\
& \left.-316 A^{3}+35 A^{4}\right) \eta^{11}+\frac{B^{2} R^{4}}{1037836800}(-8752 \\
& \left.+11592 A-4917 A^{2}+665 A^{3}\right) \eta^{13} \\
& +\frac{B^{3} R^{4}}{326918592000}\left(121474-95159 A+17641 A^{2}\right) \\
& \cdot \eta^{15}+\frac{B^{4} R^{4}}{14820309504000}(-83432+29159 A) \eta^{17} \\
& \left.+\frac{21919 B^{5} R^{4}}{844757641728000} \eta^{19}\right] .
\end{aligned}
$$

In a similar way, higher order problems solutions can be obtained. Considering the fourth-order solution,

$$
\widetilde{U}(\eta)=u_{0}(\eta)+u_{1}(\eta)+u_{2}(\eta)+u_{4}(\eta)
$$

The use of boundary conditions gives the values of unknown constants $A$ and $B$ for fixed values of $R$ in (38). For $R=0.5$, $A=1.5357$, and $B=-3.42755$, the solution is represented as follows:

$$
\begin{aligned}
& \widetilde{U}(\eta)=\left[1.5357 \eta-0.57125 \eta^{3}+0.0351938 \eta^{5}\right. \\
& +5.81905 \times 10^{-4} \eta^{7}-2.20697 \times 10^{-4} \eta^{9}-5.93588 \\
& \quad \times 10^{-7} \eta^{11}+2.03112 \times 10^{-6} \eta^{13}-4.72306 \\
& \quad \times 10^{-8} \eta^{15}-2.24977 \times 10^{-8} \eta^{17}-7.67169 \\
& \left.\quad \times 10^{-10} \eta^{19}\right] .
\end{aligned}
$$

The residual error of the problem is

Res. Error

$$
=\frac{\mathrm{d}^{4} \tilde{u}(\eta)}{\mathrm{d} \eta^{4}}+R\left[(\eta-\widetilde{u}(\eta)) \frac{\mathrm{d}^{3} \widetilde{u}(\eta)}{\mathrm{d} \eta^{3}}+3 \frac{\mathrm{d}^{2} \widetilde{u}(\eta)}{\mathrm{d} \eta^{2}}\right] .
$$

\section{Results and Discussion}

In this article, an unsteady axisymmetric flow of incompressible Newtonian fluid squeezed between two circular plates is considered. The resulting nonlinear boundary value problem is solved analytically with HPLM and HPM and numerically with ERK4.

Tables 1, 2, and 3 present the comparison of ERK4, HPM, OHAM, and HPLM solutions along with absolute residual errors for fixed values of $R$. These tables indicate that results from the proposed scheme are in good agreement with ERK4. Furthermore, Table 4 represents the comparison of RPM and HPLM solutions and residual errors for fixed values of $R$. All the tables demonstrate that results obtained using HPLM are better in terms of accuracy as compared 
TABLE 1: Comparison of solutions along with absolute residual errors for various analytical and numerical schemes at $R=0.1$.

\begin{tabular}{lcccccccc}
\hline & \multicolumn{2}{c}{ ERK4 } & \multicolumn{2}{c}{ HPM } & \multicolumn{2}{c}{ OHAM [9] } & \multicolumn{2}{c}{ HPLM } \\
& Solution & Error & Solution & Error & Solution & Error & Solution & Error \\
\hline 0.0 & 0. & $1.738 \times 10^{-5}$ & 0. & 0. & 0. & 0. & 0. & 0. \\
0.1 & 0.150158 & $5.21981 \times 10^{-8}$ & 0.150158 & $3.15139 \times 10^{-9}$ & 0.150158 & $5.37334 \times 10^{-11}$ & 0.150158 & $1.38778 \times 10^{-17}$ \\
0.2 & 0.297237 & $2.69705 \times 10^{-9}$ & 0.297237 & $7.73447 \times 10^{-9}$ & 0.297237 & $3.14675 \times 10^{-10}$ & 0.297237 & $2.22045 \times 10^{-16}$ \\
0.3 & 0.43817 & $6.72096 \times 10^{-12}$ & 0.43817 & $1.43931 \times 10^{-8}$ & 0.43817 & $8.51847 \times 10^{-10}$ & 0.43817 & $2.59792 \times 10^{-14}$ \\
0.4 & 0.5699 & $2.38437 \times 10^{-10}$ & 0.5699 & $2.22904 \times 10^{-8}$ & 0.5699 & $1.51431 \times 10^{-9}$ & 0.5699 & $7.84595 \times 10^{-13}$ \\
0.5 & 0.689397 & $2.77365 \times 10^{-11}$ & 0.689397 & $2.87631 \times 10^{-8}$ & 0.689397 & $1.90824 \times 10^{-9}$ & 0.689397 & $1.21924 \times 10^{-11}$ \\
0.6 & 0.793661 & $2.2498 \times 10^{-10}$ & 0.793661 & $2.97708 \times 10^{-8}$ & 0.793661 & $1.33301 \times 10^{-9}$ & 0.793661 & $1.22513 \times 10^{-10}$ \\
0.7 & 0.879734 & $3.20811 \times 10^{-11}$ & 0.879734 & $2.15887 \times 10^{-8}$ & 0.879734 & $1.59255 \times 10^{-9}$ & 0.879734 & $9.03036 \times 10^{-10}$ \\
0.8 & 0.944705 & $1.47457 \times 10^{-9}$ & 0.944705 & $3.64203 \times 10^{-9}$ & 0.944705 & $1.02814 \times 10^{-8}$ & 0.944705 & $5.27872 \times 10^{-9}$ \\
0.9 & 0.985722 & $2.06494 \times 10^{-8}$ & 0.985722 & $1.89627 \times 10^{-8}$ & 0.985722 & $3.446 \times 10^{-8}$ & 0.985722 & $2.57696 \times 10^{-8}$ \\
1.0 & 1. & $5.10685 \times 10^{-6}$ & 1. & $3.634 \times 10^{-8}$ & 1. & $1.03509 \times 10^{-7}$ & 1. & $1.08915 \times 10^{-7}$ \\
\hline
\end{tabular}

TABLE 2: Comparison of solutions along with absolute residual errors for various analytical and numerical schemes at $R=0.3$.

\begin{tabular}{ccccccccc}
\hline$\eta$ & \multicolumn{2}{c}{ ERK4 } & \multicolumn{2}{c}{ HPM } & \multicolumn{2}{c}{ OHAM [9] } & \multicolumn{2}{c}{ HPLM } \\
& Solution & Error & Solution & Error & Solution & Error & Solution & Error \\
\hline 0.0 & 0. & $8.41642 \times 10^{-5}$ & 0. & 0. & 0. & 0. & 0. \\
0.1 & 0.151534 & $1.80166 \times 10^{-7}$ & 0.151534 & $7.29078 \times 10^{-7}$ & 0.151534 & $6.69283 \times 10^{-9}$ & 0.151534 & $8.32667 \times 10^{-17}$ \\
0.2 & 0.299827 & $9.1271 \times 10^{-9}$ & 0.299827 & $1.82337 \times 10^{-6}$ & 0.299827 & $1.66025 \times 10^{-9}$ & 0.299827 & $6.56142 \times 10^{-14}$ \\
0.3 & 0.441661 & $8.47544 \times 10^{-10}$ & 0.441661 & $3.45076 \times 10^{-6}$ & 0.441661 & $2.33504 \times 10^{-8}$ & 0.441661 & $6.29419 \times 10^{-12}$ \\
0.4 & 0.573869 & $2.57624 \times 10^{-10}$ & 0.573869 & $5.40798 \times 10^{-6}$ & 0.573869 & $3.93945 \times 10^{-8}$ & 0.573869 & $1.94411 \times 10^{-10}$ \\
0.5 & 0.693354 & $3.69274 \times 10^{-10}$ & 0.693354 & $7.03344 \times 10^{-6}$ & 0.693354 & $2.81614 \times 10^{-8}$ & 0.693354 & $3.09131 \times 10^{-9}$ \\
0.6 & 0.797122 & $2.72398 \times 10^{-10}$ & 0.797122 & $7.32001 \times 10^{-6}$ & 0.797122 & $3.19654 \times 10^{-8}$ & 0.797122 & $3.16575 \times 10^{-8}$ \\
0.7 & 0.8823 & $5.73807 \times 10^{-10}$ & 0.882299 & $5.33901 \times 10^{-6}$ & 0.8823 & $2.21911 \times 10^{-7}$ & 0.8823 & $2.36911 \times 10^{-7}$ \\
0.8 & 0.946167 & $1.05654 \times 10^{-8}$ & 0.946167 & $9.47132 \times 10^{-7}$ & 0.946167 & $8.84707 \times 10^{-7}$ & 0.946167 & $1.40163 \times 10^{-6}$ \\
0.9 & 0.98618 & $2.05649 \times 10^{-7}$ & 0.98618 & $4.58911 \times 10^{-6}$ & 0.98618 & $3.21268 \times 10^{-6}$ & 0.98618 & $6.9077 \times 10^{-6}$ \\
1.0 & 1. & $9.11383 \times 10^{-5}$ & 1. & $8.83062 \times 10^{-6}$ & 1. & $1.11826 \times 10^{-5}$ & 1. & $2.94117 \times 10^{-5}$ \\
\hline
\end{tabular}

TABLE 3: Comparison of solutions along with absolute residual errors for various analytical and numerical schemes at $R=0.5$.

\begin{tabular}{lcccccccc}
\hline & \multicolumn{2}{c}{ ERK4 } & \multicolumn{2}{c}{ HPM } & \multicolumn{2}{c}{ OHAM [9] } & \multicolumn{2}{c}{ HPLM } \\
& Solution & Error & Solution & Error & Solution & Error & Solution & Error \\
\hline 0.0 & 0. & $2.2121 \times 10^{-4}$ & 0. & 0. & 0. & 0. & 0. \\
0.1 & 0.152999 & $4.70847 \times 10^{-7}$ & 0.152999 & $8.88098 \times 10^{-6}$ & 0.152999 & $6.23412 \times 10^{-8}$ & 0.152999 & $6.66134 \times 10^{-16}$ \\
0.2 & 0.302582 & $2.32143 \times 10^{-8}$ & 0.302582 & $2.26925 \times 10^{-5}$ & 0.302582 & $6.58388 \times 10^{-8}$ & 0.302582 & $8.07576 \times 10^{-13}$ \\
0.3 & 0.445373 & $2.03466 \times 10^{-9}$ & 0.445372 & $4.37481 \times 10^{-5}$ & 0.445373 & $3.42092 \times 10^{-7}$ & 0.445373 & $7.9418 \times 10^{-11}$ \\
0.4 & 0.578082 & $3.71427 \times 10^{-10}$ & 0.578082 & $6.94338 \times 10^{-5}$ & 0.578082 & $4.93942 \times 10^{-7}$ & 0.578082 & $2.53204 \times 10^{-9}$ \\
0.5 & 0.697548 & $9.9817 \times 10^{-10}$ & 0.697548 & $9.10469 \times 10^{-5}$ & 0.697548 & $2.42092 \times 10^{-7}$ & 0.697548 & $4.13683 \times 10^{-8}$ \\
0.6 & 0.80078 & $1.14274 \times 10^{-9}$ & 0.80078 & $9.52928 \times 10^{-5}$ & 0.80078 & $6.53046 \times 10^{-7}$ & 0.80078 & $4.32733 \times 10^{-7}$ \\
0.7 & 0.885004 & $2.15117 \times 10^{-9}$ & 0.885004 & $6.99109 \times 10^{-5}$ & 0.885004 & $3.23415 \times 10^{-6}$ & 0.885004 & $3.29216 \times 10^{-6}$ \\
0.8 & 0.947702 & $2.92081 \times 10^{-8}$ & 0.947702 & $1.30127 \times 10^{-5}$ & 0.947702 & $1.2206 \times 10^{-5}$ & 0.947702 & $1.97295 \times 10^{-5}$ \\
0.9 & 0.986659 & $5.72395 \times 10^{-7}$ & 0.986659 & $5.87658 \times 10^{-5}$ & 0.986659 & $4.41008 \times 10^{-5}$ & 0.986659 & $9.82181 \times 10^{-5}$ \\
1.0 & 1. & $2.53597 \times 10^{-4}$ & 1. & $1.13563 \times 10^{-4}$ & 1. & $1.54022 \times 10^{-4}$ & 1. & $4.21474 \times 10^{-4}$ \\
\hline
\end{tabular}


TABLE 4: Comparison of HPLM and RPM solutions along with absolute residual errors for various $R$.

\begin{tabular}{lcccccccc}
\hline & \multicolumn{3}{c}{$R=0.01$} & \multicolumn{2}{c}{$R=0.05$} \\
$\eta$ & \multicolumn{2}{c}{ RPM [1] } & \multicolumn{2}{c}{ HPLM } & \multicolumn{2}{c}{ RPM [1] } & \multicolumn{2}{c}{ HPLM } \\
& Solution & Error & Solution & Error & Solution & Error & Solution & Error \\
\hline 0.0 & 0. & $2.56192 \times 10^{-8}$ & 0. & 0. & 0. & 0. & 0. & 0. \\
0.1 & 0.149565 & $5.12309 \times 10^{-8}$ & 0.149565 & $5.11534 \times 10^{-13}$ & 0.149826 & $3.1947 \times 10^{-6}$ & 0.149826 & $6.26892 \times 10^{-11}$ \\
0.2 & 0.296122 & $7.5716 \times 10^{-8}$ & 0.296122 & $2.08073 \times 10^{-11}$ & 0.296614 & $6.39202 \times 10^{-6}$ & 0.296614 & $2.56895 \times 10^{-9}$ \\
0.3 & 0.436665 & $9.5916 \times 10^{-8}$ & 0.436665 & $2.1618 \times 10^{-10}$ & 0.437329 & $9.4544 \times 10^{-6}$ & 0.437329 & $2.68881 \times 10^{-8}$ \\
0.4 & 0.568188 & $1.06146 \times 10^{-7}$ & 0.568188 & $1.27207 \times 10^{-9}$ & 0.568943 & $1.1987 \times 10^{-5}$ & 0.568943 & $1.5913 \times 10^{-7}$ \\
0.5 & 0.687687 & $9.85655 \times 10^{-8}$ & 0.687687 & $5.39369 \times 10^{-9}$ & 0.688442 & $1.32764 \times 10^{-5}$ & 0.688442 & $6.77516 \times 10^{-7}$ \\
0.6 & 0.792164 & $6.50776 \times 10^{-8}$ & 0.792164 & $1.83847 \times 10^{-8}$ & 0.792825 & $1.2338 \times 10^{-5}$ & 0.792825 & $2.31613 \times 10^{-6}$ \\
0.7 & 0.878622 & $1.76889 \times 10^{-9}$ & 0.878622 & $5.35595 \times 10^{-8}$ & 0.879113 & $8.15489 \times 10^{-6}$ & 0.879113 & $6.76161 \times 10^{-6}$ \\
0.8 & 0.94407 & $8.25984 \times 10^{-8}$ & 0.94407 & $1.38591 \times 10^{-7}$ & 0.94435 & $2.35963 \times 10^{-7}$ & 0.94435 & $1.75223 \times 10^{-5}$ \\
0.9 & 0.985522 & $1.51911 \times 10^{-7}$ & 0.985522 & $3.26876 \times 10^{-7}$ & 0.98561 & $1.03197 \times 10^{-5}$ & 0.98561 & $4.13704 \times 10^{-5}$ \\
1.0 & 1. & $2.53597 \times 10^{-4}$ & 1. & $7.1565 \times 10^{-7}$ & 1. & $1.89889 \times 10^{-5}$ & 1. & $9.06357 \times 10^{-5}$ \\
\hline
\end{tabular}

TABLE 5: Various order HPLM solutions along with absolute residual errors.

\begin{tabular}{|c|c|c|c|c|c|c|}
\hline \multirow{2}{*}{$\eta$} & \multicolumn{2}{|c|}{ 0th-order } & \multicolumn{2}{|c|}{ 2nd-order } & \multicolumn{2}{|c|}{4 th-order } \\
\hline & Solution & Error & Solution & Error & Solution & Error \\
\hline 0.0 & 0 . & 0 . & 0. & $4.88607 \times 10^{-10}$ & 0. & 0 . \\
\hline 0.1 & 0.1495 & 0.07515 & 0.015015 & $2.0221 \times 10^{-8}$ & 0.150158 & $1.38778 \times 10^{-17}$ \\
\hline 0.2 & 0.296 & 0.1512 & 0.297237 & $2.13694 \times 10^{-7}$ & 0.297237 & $2.22045 \times 10^{-16}$ \\
\hline 0.3 & 0.4365 & 0.22905 & 0.43817 & $1.27408 \times 10^{-6}$ & 0.43817 & $2.59792 \times 10^{-14}$ \\
\hline 0.4 & 0.568 & 0.3096 & 0.5699 & $5.45323 \times 10^{-6}$ & 0.5699 & $7.84595 \times 10^{-13}$ \\
\hline 0.5 & 0.6875 & 0.39375 & 0.689397 & $1.87166 \times 10^{-5}$ & 0.689397 & $1.21924 \times 10^{-11}$ \\
\hline 0.6 & 0.792 & 0.4824 & 0.793661 & $5.477 \times 10^{-5}$ & 0.793661 & $1.22513 \times 10^{-10}$ \\
\hline 0.7 & 0.8785 & 0.57645 & 0.879734 & $1.42199 \times 10^{-4}$ & 0.879734 & $9.03036 \times 10^{-10}$ \\
\hline 0.8 & 0.944 & 0.6768 & 0.944705 & $3.36171 \times 10^{-4}$ & 0.944705 & $5.27872 \times 10^{-9}$ \\
\hline 0.9 & 0.9855 & 0.78435 & 0.985722 & $7.37122 \times 10^{-4}$ & 0.985722 & $2.57696 \times 10^{-8}$ \\
\hline 1.0 & 1. & 0.9 & 1. & $7.96168 \times 10^{-6}$ & 1. & $1.08915 \times 10^{-7}$ \\
\hline
\end{tabular}

TABLE 6: Comparison of HPLM and ERK4 solutions.

\begin{tabular}{cccc}
\hline$\eta$ & & $\mid$ HPLM - ERK4 & \\
& $R=0.1$ & $R=0.3$ & $R=0.5$ \\
\hline 0.0 & 0. & 0. & 0. \\
0.1 & $1.20703 \times 10^{-12}$ & $3.30592 \times 10^{-10}$ & $4.7981 \times 10^{-9}$ \\
0.2 & $2.33102 \times 10^{-12}$ & $6.37707 \times 10^{-10}$ & $9.24961 \times 10^{-9}$ \\
0.3 & $3.2877 \times 10^{-12}$ & $8.98009 \times 10^{-10}$ & $1.30114 \times 10^{-8}$ \\
0.4 & $3.99419 \times 10^{-12}$ & $1.08846 \times 10^{-9}$ & $1.57475 \times 10^{-8}$ \\
0.5 & $4.36812 \times 10^{-12}$ & $1.18646 \times 10^{-9}$ & $1.71329 \times 10^{-8}$ \\
0.6 & $4.32793 \times 10^{-12}$ & $1.17012 \times 10^{-9}$ & $1.68584 \times 10^{-8}$ \\
0.7 & $3.79589 \times 10^{-12}$ & $1.01905 \times 10^{-9}$ & $1.46445 \times 10^{-8}$ \\
0.8 & $2.71873 \times 10^{-12}$ & $7.19961 \times 10^{-10}$ & $1.03195 \times 10^{-8}$ \\
0.9 & $1.19406 \times 10^{-12}$ & $3.00204 \times 10^{-10}$ & $4.29345 \times 10^{-9}$ \\
1.0 & $1.1395 \times 10^{-13}$ & $1.44055 \times 10^{-16}$ & $1.83218 \times 10^{-16}$ \\
\hline
\end{tabular}

to other stated schemes. In addition to the above-mentioned tables, Table 5 shows various order solutions along with absolute residual errors and confirms the convergence of HPLM solution. Moreover, Table 6 indicates the comparison of HPLM solution with numerical (ERK4) solutions. It shows that HPLM results are in very good agreement with ERK4.

Furthermore, Figure 2 indicates the residual errors of HPLM, OHAM, HPM, and ERK4 for $R=0.5$. In order to capture more details, the figure also shows an inset at a more finer resolution showing the residual errors between HPLM, OHAM, and ERK4. Figure 3 presents the comparison of second-order absolute residual errors between HPLM and RPM. The convergence of HPLM solution is shown in Figure 4, where the average absolute residual errors against various order solutions are presented.

Figure 5 demonstrates the pressure variation for various values of $R$. It is observed that when the plates approach each other the pressure at the plates is higher than that at the centre and vice versa.

Figures 6-9 show the effect of positive and negative values of Reynolds number $R$ on the velocity profiles. The effect of 


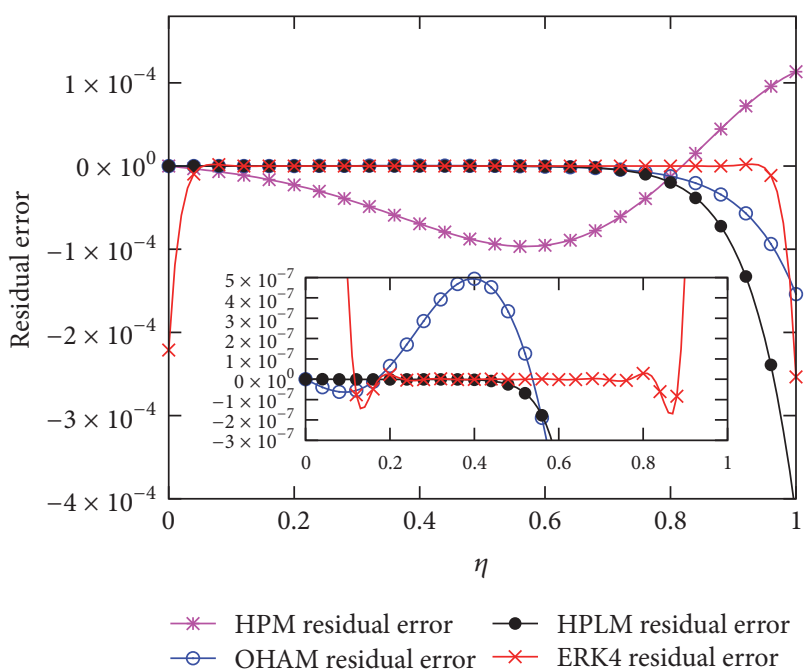

FIgURE 2: Residual error of HPM, OHAM, HPLM, and ERK4 for Reynolds number $R=0.5$. In the inset, a more finer resolution of the residual error of OHAM, HPLM, and ERK4 is shown.

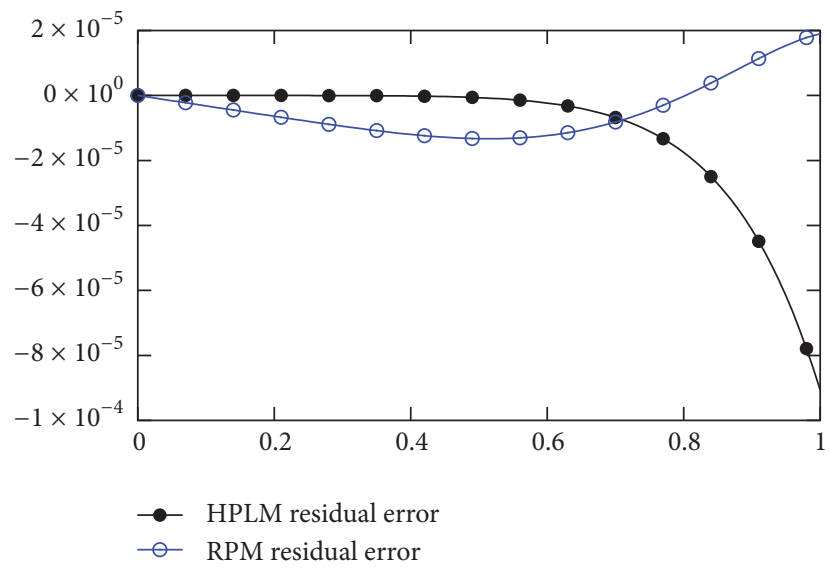

FIgURE 3: Comparison of residual errors of HPLM and RPM for Reynolds number $R=0.05$.

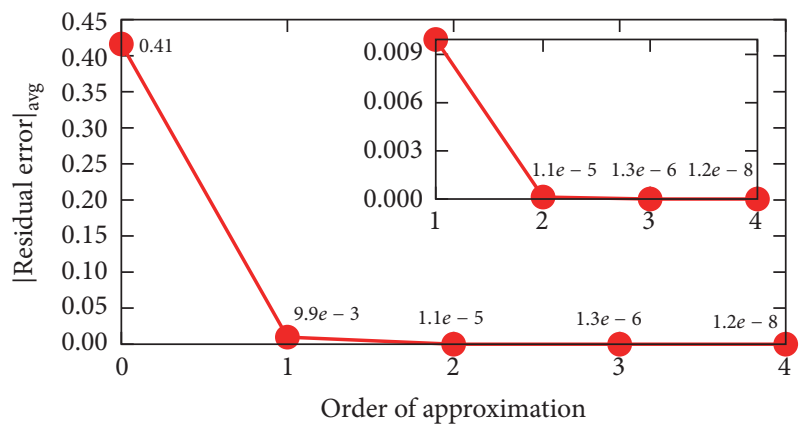

FIgURE 4: Convergence of HPLM.

negative $R$ shows an opposite effect on the velocity profile as compared to positive $R$.

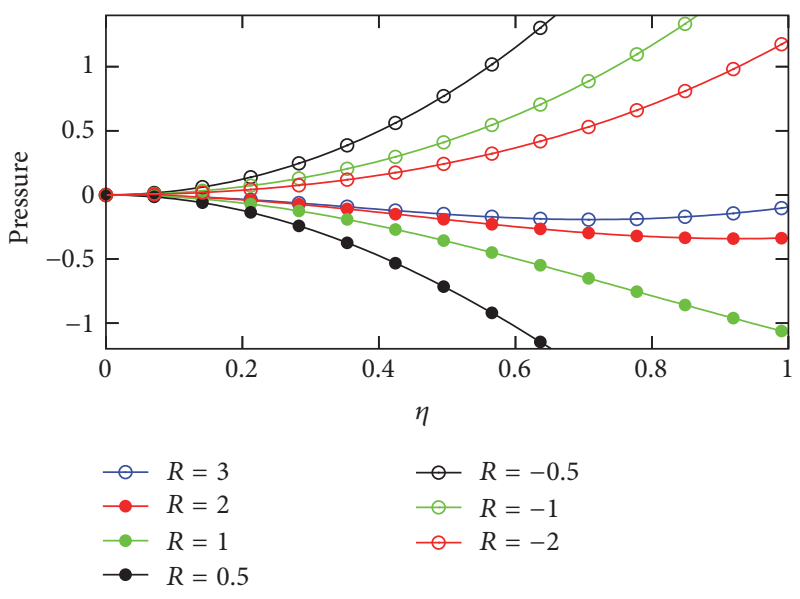

FIgURE 5: Pressure variation with $\eta$.

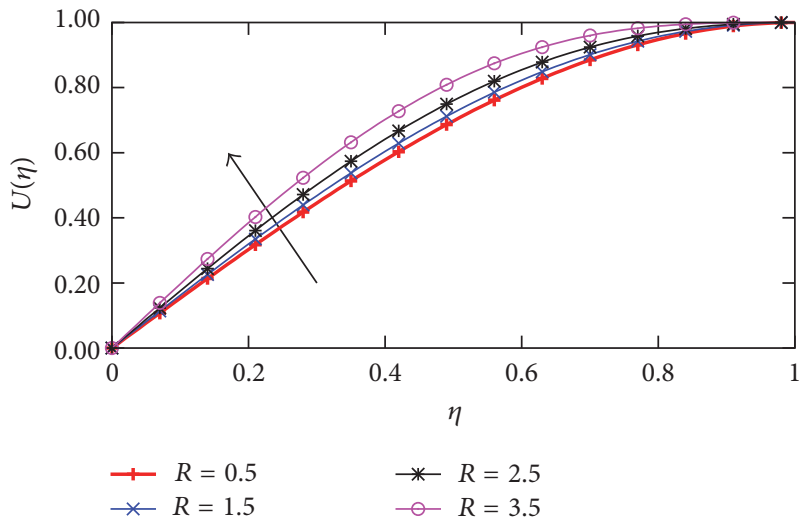

FIGURE 6: Effect of positive Reynolds number $R$ on the normal velocity.

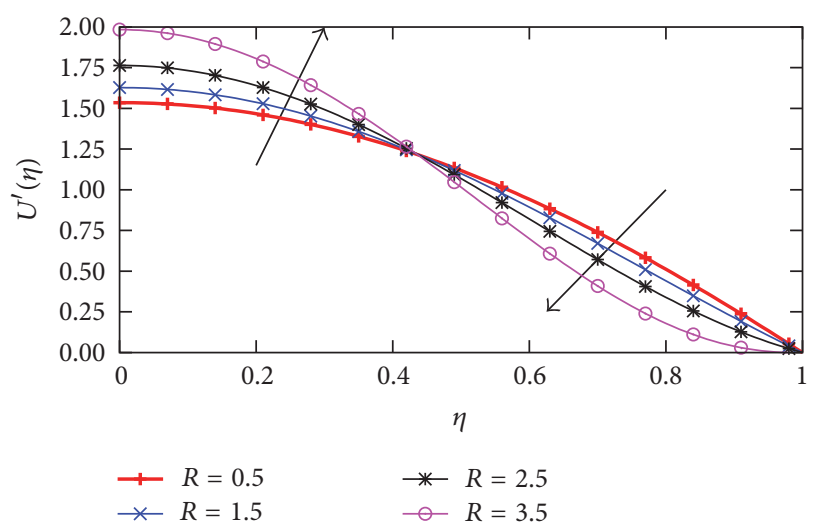

FIGURE 7: Effect of positive Reynolds number $R$ on the longitudinal velocity.

\section{Conclusion}

A similarity solution for an unsteady axisymmetric squeezing flow is obtained using a modification of the HPM named as Homotopy Perturbation Laplace Method (HPLM). Analysis of the residual errors confirms that HPLM is an efficient 


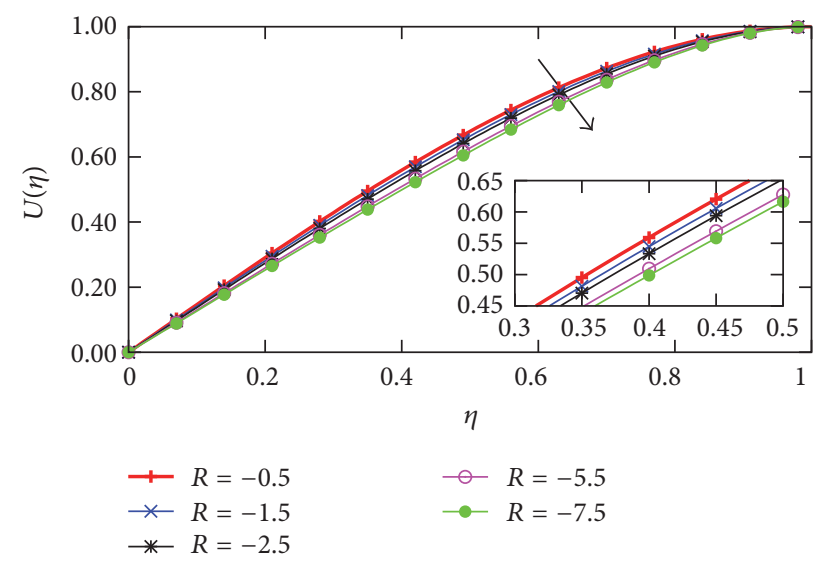

FIGURE 8: Effect of negative Reynolds number $R$ on the normal velocity.

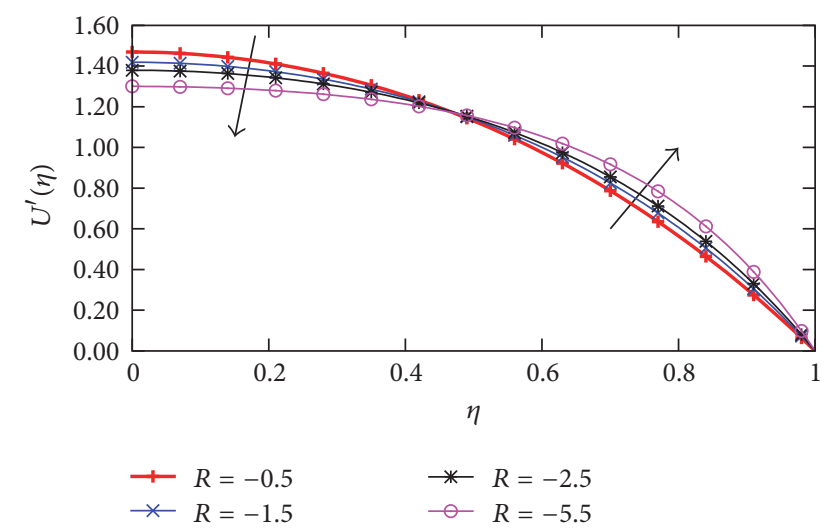

FIGURE 9: Effect of negative Reynolds number $R$ on the longitudinal velocity.

scheme as compared to other techniques presented in this article. The convergence and validity of the proposed HPLM scheme is verified by means of residual error and compared with numerical solutions. The analysis of obtained result shows that HPLM can be effectively used in various fields of science and technology as it gives improved results in terms of accuracy.

\section{Conflicts of Interest}

The authors declare that they have no conflicts of interest.

\section{References}

[1] P. S. Gupta and A. S. Gupta, "Squeezing flow between parallel plates," Wear, vol. 45, no. 2, pp. 177-185, 1977.

[2] R. Törnqvist, P. Sunderland, and J. A. E. Månson, "Nonisothermal process rheology of thermoplastic composites for compression flow moulding," Composites Part A: Applied Science and Manufacturing, vol. 31, no. 9, pp. 917-927, 2000.

[3] P. Shirodkar, A. Bravo, and S. Middleman, "Lubrication flows in viscoelastic liquids: 2. Effect of slip on squeezing flow between approaching parallel rigid planes," Chemical Engineering Communications, vol. 14, no. 3-6, pp. 151-175, 1982.

[4] Y. Tian, S. Wen, and Y. Meng, "Compressions of electrorheological fluids under different initial gap distances," Physical Review E, vol. 67, no. 5, Article ID 051501, 2003.

[5] R. B. Bird, R. C. Armstrong, and O. Hassager, Dynamics of Polymeric Liquids. Vol. 1: Fluid Mechanics, 1987.

[6] J. L. Kokini, J. B. Kadane, and E. L. Cussler, "Liquid texture perceived in the mouth," Journal of Texture Studies, vol. 8, no. 2, pp. 195-218, 1977.

[7] J.-H. He, "Homotopy perturbation technique," Computer Methods in Applied Mechanics and Engineering, vol. 178, no. 3-4, pp. 257-262, 1999.

[8] M. Qayyum, H. Khan, M. T. Rahim, and I. Ullah, "Modeling and analysis of unsteady axisymmetric squeezing fluid flow through porous medium channel with slip boundary," PLoS ONE, vol. 10, no. 3, Article ID e0117368, 2015.

[9] M. Qayyum, H. Khan, M. T. Rahim, and I. Ullah, "Analysis of unsteady axisymmetric squeezing fluid flow with slip and no-slip boundaries using OHAM," Mathematical Problems in Engineering, vol. 2015, Article ID 860857, 11 pages, 2015.

[10] A. Qayyum, M. Awais, A. Alsaedi, and T. Hayat, "Unsteady squeezing flow of jeffery fluid between two parallel disks," Chinese Physics Letters, vol. 29, no. 3, Article ID 034701, 2012.

[11] H. M. Laun, M. Rady, and O. Hassager, "Analytical solutions for squeeze flow with partial wall slip," Journal of Non-Newtonian Fluid Mechanics, vol. 81, no. 1-2, pp. 1-15, 1999.

[12] W.-P. Breugem, "The effective viscosity of a channel-type porous medium," Physics of Fluids, vol. 19, no. 10, Article ID 103104, 2007.

[13] A. M. Siddiqui, M. Ahmed, and Q. K. Ghori, "Couette and Poiseuille flows for non-Newtonian fluids," International Journal of Nonlinear Sciences and Numerical Simulation, vol. 7, no. 1, pp. 15-26, 2006.

[14] A. M. Siddiqui, R. Mahmood, and Q. K. Ghori, “Thin film flow of a third grade fluid on a moving belt by He's homotopy perturbation method," International Journal of Nonlinear Sciences and Numerical Simulation, vol. 7, no. 1, pp. 7-14, 2006.

[15] T. Kajishima and K. Taira, "Finite-difference discretization of the advection-diffusion equation," in Computational Fluid Dynamics, pp. 23-72, Springer Nature, 2016.

[16] R. Ellahi, E. Shivanian, S. Abbasbandy, and T. Hayat, "Numerical study of magnetohydrodynamics generalized Couette flow of Eyring-Powell fluid with heat transfer and slip condition," International Journal of Numerical Methods for Heat \& Fluid Flow, vol. 26, no. 5, pp. 1433-1445, 2016.

[17] J.-H. He, "A coupling method of a homotopy technique and a perturbation technique for non-linear problems," International Journal of Non-Linear Mechanics, vol. 35, no. 1, pp. 37-43, 2000.

[18] J.-H. He, "Homotopy perturbation method: a new nonlinear analytical technique," Applied Mathematics and Computation, vol. 135, no. 1, pp. 73-79, 2003.

[19] J.-H. He, "Homotopy perturbation method for solving boundary value problems," Physics Letters. A, vol. 350, no. 1-2, pp. 87$88,2006$.

[20] N. Herişanu and V. Marinca, "Optimal homotopy perturbation method for a non-conservative dynamical system of a rotating electrical machine," Zeitschrift fur Naturforschung. Section A Journal of Physical Sciences, vol. 67, no. 8-9, pp. 509-516, 2012.

[21] F. Wang, W. Li, and H. Zhang, "A new extended homotopy perturbation method for nonlinear differential equations," 
Mathematical and Computer Modelling, vol. 55, no. 3-4, pp. 1471-1477, 2012.

[22] A. Nazari-Golshan, S. S. Nourazar, H. Ghafoori-Fard, A. Yildirim, and A. Campo, "A modified homotopy perturbation method coupled with the Fourier transform for nonlinear and singular Lane-Emden equations," Applied Mathematics Letters, vol. 26, no. 10, pp. 1018-1025, 2013.

[23] S. S. Nourazar and A. Nazari-Golshan, "A new modification to homotopy perturbation method combined with Fourier transform for solving nonlinear Cauchy reaction diffusion equation," Indian Journal of Physics, vol. 89, no. 1, pp. 61-71, 2015.

[24] D. D. Ganji, A. R. Sahouli, and M. Famouri, "A new modification of He's homotopy perturbation method for rapid convergence of nonlinear undamped oscillators," Journal of Applied Mathematics and Computing, vol. 30, no. 1-2, pp. 181-192, 2009.

[25] A. A. Hemeda, "Modified homotopy perturbation method for solving fractional differential equations," Journal of Applied Mathematics, vol. 2014, Article ID 594245, 9 pages, 2014. 

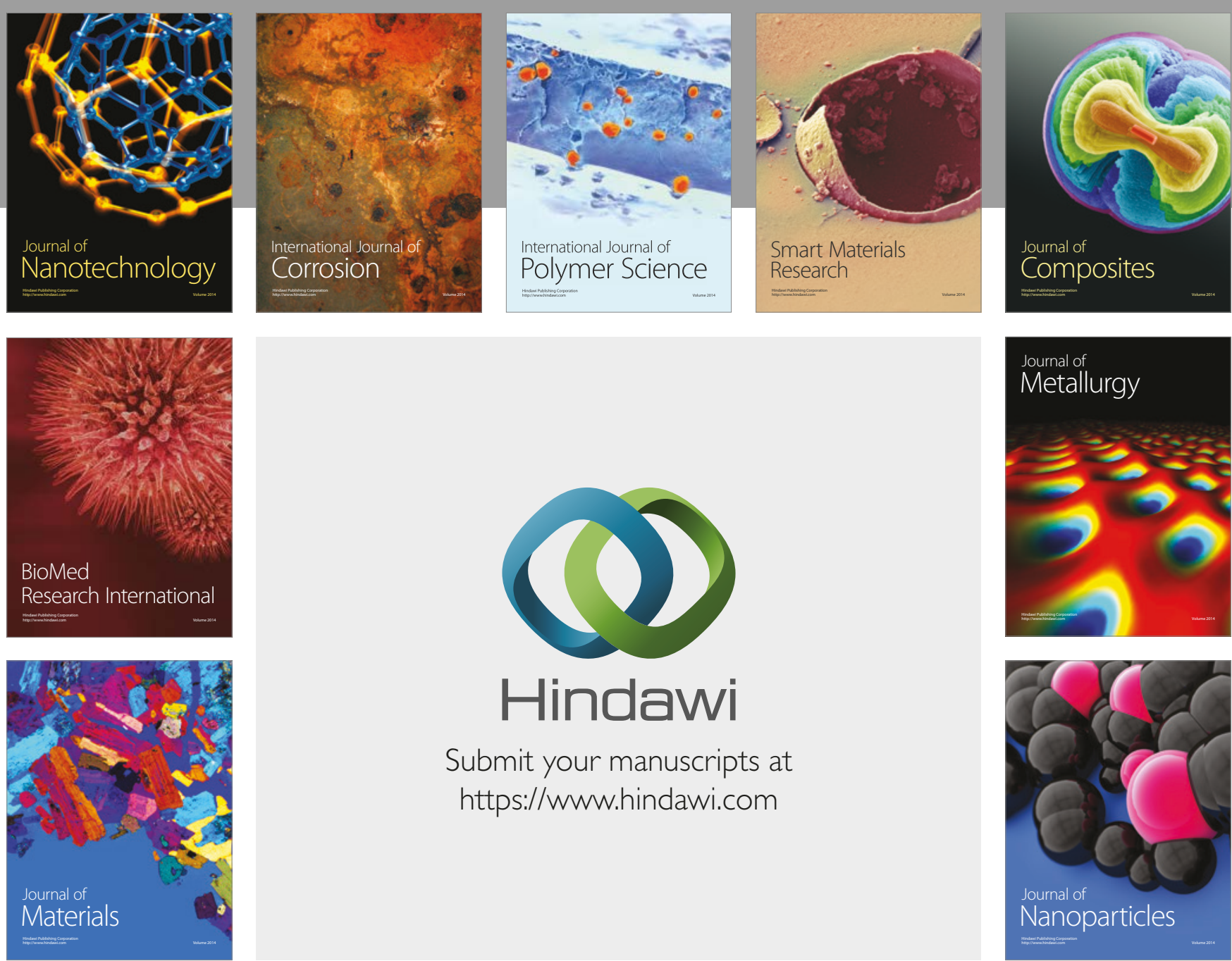

\section{Hindawi}

Submit your manuscripts at

https://www.hindawi.com
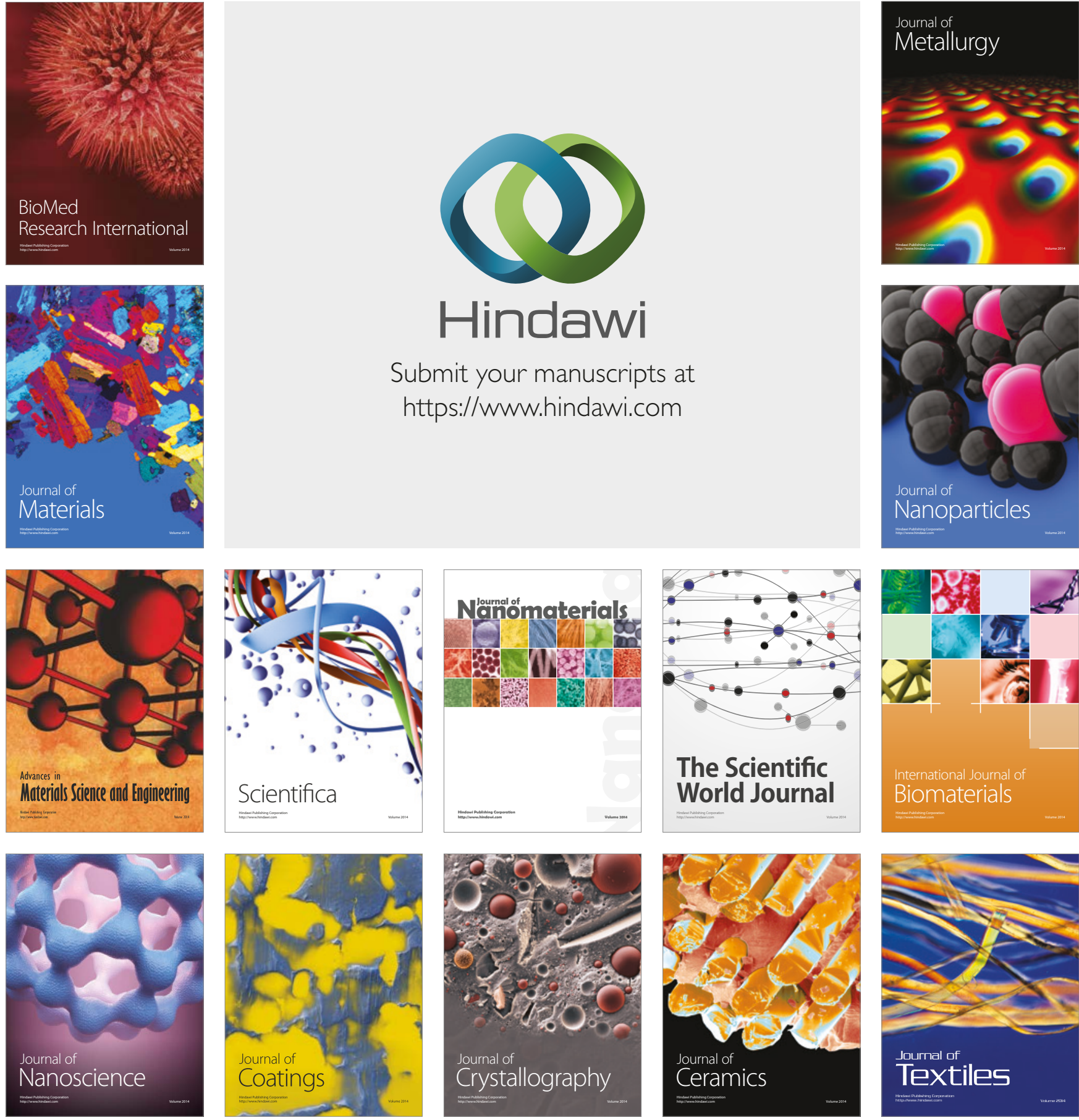

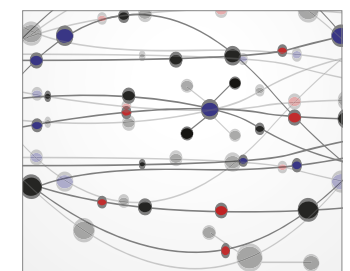

The Scientific World Journal
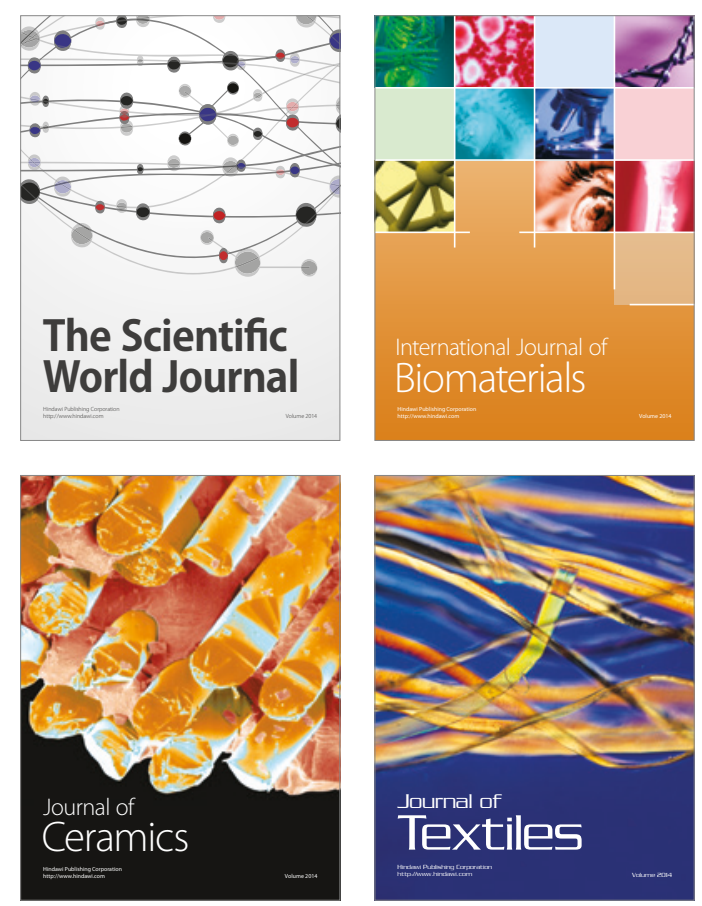